\title{
LncRNA SNHG4 Attenuates Inflammatory Responses by Sponging miR-449c-5p and Up-Regulating STAT6 in Microglial During Cerebral Ischemia-Reperfusion Injury
}

This article was published in the following Dove Press journal:

Drug Design, Development and Therapy

\author{
Shuo Zhang ${ }^{1,2}$ \\ Wen-chong Sun ${ }^{2}$ \\ Zuo-di Liang ${ }^{2}$ \\ Xiu-ru Yin ${ }^{2}$ \\ Zhen-rong $\mathrm{Ji}^{2}$ \\ Xiao-huan Chen ${ }^{2}$ \\ Min-jie Wei (DD) \\ Ling $\mathrm{Pei}^{2}$
}

'Department of Pharmacology, School of Pharmacy, China Medical University, Shenyang, Liaoning II 000I, People's Republic of China; ${ }^{2}$ Department of Anesthesiology, The First Affiliated Hospital of China Medical University, Shenyang, Liaoning I I000I, People's Republic of China
Correspondence: Min-jie Wei; Ling Pei Email koxu078nzod@I63.com;

lingpei49@sina.com
Background: Inflammatory response mediated by microglia plays a key role in cerebral ischemia-reperfusion injury. This study intends to probe the role of lncRNA SNHG4 in regulating the inflammatory response of the microglia during cerebral ischemia reperfusion. Materials and Methods: Blood samples and cerebrospinal fluid samples were collected from acute cerebral infarction (ACI) patients and healthy controls. The middle cerebral artery occlusion (MCAO) models were constructed with rats. LPS induction and oxygen-glucose deprivation methods were respectively applied to simulate the activation of microglia in vitro. qRT-PCR was employed to determine the expressions of SNHG4, miR-449c-5p and related inflammatory factors in vivo and in vitro. The inflammatory responses of the microglia subject to the varied expressions of SNHG4 and miR-449c-5p were detected. Luciferase assays were conducted to verify the crosstalk involving SNHG4, miR-449c-5p and STAT6.

Results: Compared with the control group, the expression of SNHG4 derived from the samples of ACI patients and the microglia of MCAO group were remarkably downregulated, but the expression of $\operatorname{miR}-449 \mathrm{c}-5 \mathrm{p}$ was dramatically up-regulated. Overexpression of SNHG4 and knock-down of miR-449c-5p could inhibit the expression of pro-inflammatory cytokine in the microglia and promote the expression of antiinflammatory factors. Meanwhile, the phospho-STAT6 was up-regulated, whereas the knockdown of SNHG4 and over-expression of miR-449c-5p in microglia had the opposite effects. Luciferase assay confirmed that SNHG4 could target miR-449c-5p, while miR-449c-5p could target STAT6.

Conclusion: SNHG4 can regulate STAT6 and repress inflammation by adsorbing miR$449 \mathrm{c}-5 \mathrm{p}$ in microglia during cerebral ischemia-reperfusion injury.

Keywords: SNHG4, STAT6, miR-449c-5p, ischemia-reperfusion injury

\section{Introduction}

Stroke is the second major cause of mortality and disability worldwide, leading to a range of secondary diseases, including cognitive impairment, epilepsy and so on. ${ }^{1}$ Ischemic stroke is the most typical stroke, which is caused by the lack of blood flowing to the brain. ${ }^{2}$ Although the pathophysiological mechanisms of stroke have been extensively studied and diverse clinical trials have been completed, the only effective treatment depends on endovascular thrombolysis or thrombectomy. ${ }^{3}$ Due 
to the limited effective treatment strategy, it is of great urgency to seek alternative therapeutic strategies.

Ischemic stroke can trigger a variety of physiological responses after causing ischemic brain injury, such as oxidative stress, calcium and sodium overload, glutamate excitotoxicity and neuroinflammation. ${ }^{4}$ Neuroinflammation plays a very important role in the pathogenesis of ischemic brain injury and ischemia-reperfusion injury. ${ }^{5}$ And this process is mainly caused by the abnormal activation of glial cells, especially microglia, and the subsequent production of inflammatory mediators. ${ }^{6}$ Microglia are the major immune cells of the central nervous system (CNS). Physiologically, resting microglia can continuously monitor the microenvironment of the CNS. ${ }^{7}$ Once the cerebral ischemia occurs, the microglia will be activated. ${ }^{8}$ On the one hand, activated microglia migrate to the ischemic area to remove noxious substances and maintain tissue homeostasis. ${ }^{9}$ On the other hand, hyperactivated microglia produce excessive inflammatory cytokines such as tumor necrosis factor- $\alpha$ (TNF- $\alpha$ ), interleukin-1 $\beta$ (IL-1 $\beta$ ) and interleukin-6 (IL-6), which lead to uncontrolled inflammation and aggravate tissue damage and neuronal death. ${ }^{10}$ Therefore, inhibiting excessive activation and inflammatory response of microglia in the early stage of acute ischemic stroke can effectively ameliorate brain injury. ${ }^{11}$

Long non-coding RNAs (IncRNAs) are transcripts that consist of more than 200 bases in length and rarely have open reading frame (ORF). They serve the specific regulations in transcriptional, post-transcriptional, and epigenetic phases, and have highly specific expressions in the CNS, which can effectively regulate the development of the CNS and the progression of disease. ${ }^{12}$ It is reported that lncRNA FosDT facilitates ischemic brain impairment by interacting with REST-related chromatin-modifying proteins. ${ }^{13}$ In addition, it is demonstrated that IncRNA-1810034e14Rik has protective effects on the brain of mice with middle cerebral artery occlusion (MCAO), and can inhibit the activation of microglia and the release of pro-inflammatory factors so as to play an anti-inflammatory role. ${ }^{14}$ These studies suggest that lncRNA can be used as a promising target for the therapy of brain impairment after ischemic stroke.

MicroRNAs (miRNAs) are single-stranded nucleotides which are often composed of 19-22 bases in length. They can bind to the 3'-untranslated region (3'-UTR) of the target mRNA, leading to translational inhibition and mRNA degradation, so as to regulate the expression of target genes. ${ }^{15}$ miRNAs have been validated to be crucial players in ischemic stroke. ${ }^{16} \mathrm{~A}$ recent study demonstrates that the knockout of miR-497 can effectively improve the level of bcl-2/-w protein in ischemic areas, ameliorate ischemic cerebral infarction, and facilitate neurological function recovery after focal cerebral ischemia in mice. ${ }^{17}$ Additionally, studies have demonstrated that in the acute phase of stroke of adult mice, inhibition of miR-210 inhibits pro-inflammatory responses and reduces ischemic brain impairment. ${ }^{18}$

In this study, it was demonstrated that IncRNA small nucleolar RNA host gene 4 (SNHG4) was down-regulated and that miR-449c-5p was up-regulated in the blood samples and cerebrospinal fluid samples from patients with acute cerebral infarction (ACI). The results of gain- and loss-of function experiments showed that SNHG4 could regulate the expression of miR-449c-5p, and overexpression of SNHG4 could activate the STAT6 signaling pathway, regulate the activation and polarization of microglia, and attenuate the inflammation during cerebral ischemia-reperfusion injury.

\section{Materials and Methods Sample Collection}

Blood and cerebrospinal fluid samples of ACI patients and healthy controls ( $\mathrm{n}=30$ in each group) were obtained from Department of Neurosurgery, the First Affiliated Hospital of China Medical University. The samples were stored in liquid nitrogen before experiments. The study was approved by the Ethics Review Board of the First Affiliated Hospital of China Medical University. Each patient signed the informed written consent.

\section{Animal}

Sprague-Dawley (SD) rats (gender: male, body weight: 210-230 g, age: 6-8 weeks) were procured from the Animal Center of First Affiliated Hospital of China Medical University. All the animals were housed in groups and maintained in a $12 \mathrm{~h}$ light: $12 \mathrm{~h}$ dark cycle and they had easy access to water and food. All SD rats were acclimated to the environment for one week before modeling. All experiments were performed in accordance with the NIH Guide for the Care and Use of Laboratory Animals and approved by the Ethics Review Board of First Affiliated Hospital of China Medical University.

\section{Establishment of the Rat Model with Cerebral Ischemia-Reperfusion Injury (MCAO Model)}

The rats were anesthetized by intraperitoneal injection of $10 \%$ chloral hydrate $(0.35 \mathrm{~g} / \mathrm{kg})$, then the rats were fixed on 
the operating table, and the incisions were cut in the middle of the rat necks. The left common carotid arteries were isolated after the exposure of the carotid sheaths. Subsequently, the proximal common carotid arteries were ligated with the 5-0 thread, then an incision was made on common carotid artery. After that, focal cerebral ischemia was induced by occlusion of the left middle cerebral artery with a nylon monofilament (diameter criteria $0.25 \mathrm{~mm}$ ) with a heparincoated tip. The monofilament was introduced into internal carotid artery through the incision, up to the origin of the anterior cerebral artery and occluded the middle cerebral artery and anterior cerebral artery for $2 \mathrm{~h}$. The monofilament was then withdrawn to recover perfusion. After that, the reperfusion for $24 \mathrm{~h}$ was conducted. Then, the ischemic-reperfusion models were successfully established. In the sham operation group, the arteries were isolated without ligation.

\section{Cell Culture}

Rat immortal microglia cell line (HAPI cells) and human embryo kidney cell line HEK293 were provided by American Type Culture Collection (ATCC, Rockville, USA) and cells were cultured in Dulbeccos modified Eagles medium (DMEM; Invitrogen, Carlsbad, CA, USA) containing $10 \%$ fetal bovine serum (FBS) and maintained at $37^{\circ} \mathrm{C}$ in $5 \% \mathrm{CO}_{2}$. LPS induction model: microglia were treated with LPS (100 ng/mL, Escherichia coli 055: B5, Sigma, St. Louis, MO, USA) for $3 \mathrm{~h}$. Oxygen-glucose deprivation (OGD) model: the culture condition was altered from normal medium to glucose-free medium. After rinsing with $5 \% \mathrm{CO}_{2} / 95 \%$ $\mathrm{N}_{2}$ for 15 min at $2 \mathrm{psi}(1 \mathrm{psi}=6.89 \mathrm{kPA})$, the microglia cells were maintained in an anoxic chamber (Billups-Rothenberg, Del Mar, CA). The chamber was then incubated for $4 \mathrm{~h}$ at $37{ }^{\circ} \mathrm{C}$. After the end of OGD, the cells were returned to normal medium and incubated at $37^{\circ} \mathrm{C}$ in $5 \% \mathrm{CO}_{2}$ for $24 \mathrm{~h}$.

\section{Cell Transfection}

The HAPI cells were rinsed 3 times with PBS buffer and then trypsinized for $2 \mathrm{~min}$. Subsequently, the cells were suspended in serum-free medium and evenly inoculated into a 12 -well plate at $1 \times 10^{6} / \mathrm{mL}$ to reach a confluence of $50 \%$ to $60 \%$. The transfection reagent was diluted in $3 \mu \mathrm{L} / \mathrm{L}$ serum-free medium, incubated at $37{ }^{\circ} \mathrm{C}$ for $20 \mathrm{~min}$, and the reagents containing pc-DNA3.1-SNHG4, sh-SNHG4 or controls (pc-DNA3.1 or sh-Scramble) were diluted in serum-free medium, respectively, and incubated for $5 \mathrm{~min}$ at room temperature. After that, the medium was mixed withan equal volume of transfection reagent and then used to transfect the cells. After $12 \mathrm{~h}$, the state of the transfected cells was observed, and the serum-free medium was replaced with the complete medium. Then, after $48 \mathrm{~h}$ of culture, the RNAs were extracted to verify the transfection efficiency. In the same way, miR-449c-5p mimic, miR$449 \mathrm{c}-5 \mathrm{p}$ inhibitors and control group (control mimics or control inhibitors) were transfected into HAPI cells. The SNHG4 plasmid and the miR-449c-5p mimic and inhibitor were designed and synthesized by GenePharma (Shanghai, China). Cells transfections were performed with Lipofectamine $^{\circledR} 2000$ (Invitrogen, Carlsbad, CA, USA).

\section{Cell Viability Assays}

LDH cytotoxicity assay (Beyotime Biotechnology, Shanghai, China) and Cell Counting kit-8 (CCK-8) assay (Beyotime Biotechnology, Shanghai, China) were used to measure the cell viability. For LDH assay, cells were incubated with $150 \mu \mathrm{L} \mathrm{LDH}$ release reagent for $1 \mathrm{~h}$ at $37^{\circ} \mathrm{C}$. The absorbance at $490 \mathrm{~nm}$ was measured using a microplate reader (Bio-Rad, Hercules, California, USA). For CCK-8 assay, $10 \mu \mathrm{L}$ of CCK-8 solution was added to each well in incubated at $37^{\circ} \mathrm{C}$ for $2 \mathrm{~h}$. Then, the absorbance at $450 \mathrm{~nm}$ was measured with a microplate reader (Bio-Rad, Hercules, California, USA).

\section{Immunohistochemistry Assay}

Immunohistochemistry was adopted to detect the expression of caspase-3. Paraffin-embedded tissue slices were dewaxed using xylene and hydrated using a gradient of ethanol. The slices were immersed in $10 \mathrm{~mL}$ citrate buffer $(\mathrm{pH}$ 6.0) and boiled in a $121{ }^{\circ} \mathrm{C}$ pressure cooker for $4 \mathrm{~min}$ to recover antigen. The slides were then slowly cooled at room temperature, soaked in $10 \mathrm{~mL}$ citrate buffer and washed with PBS. Endogenous peroxidase activity was terminated by treatment with $3 \% \mathrm{H}_{2} \mathrm{O}_{2}$ for $15 \mathrm{~min}$ at room temperature. The slices were incubated with the antibody anti-Cleavedcaspase-3 (1:300; ab2302; Abcam) overnight at $4{ }^{\circ} \mathrm{C}$, then secondary antibody was added and incubated for $0.5 \mathrm{~h}$ at room temperature. After that, the slices were stained with 3,5-diaminobenzidine (DAB, Fuzhou, Maxim), observed and photographed under the optical microscope.

\section{Enzyme-Linked Immunosorbent Assay (ELISA)}

Following the protocols (Abcam, Shanghai, China), specific ELISA kits were used to determine the contents of proinflammatory cytokines TNF- $\alpha$, IL-1 $\beta$ and IL-6 and 
anti-inflammatory factors IL-4, IL-10 and TGF- $\beta$ in cell culture supernatant and brain tissue homogenate. The tissue and saline were added to the homogenizer, thoroughly mixed and pulverized to obtain tissue homogenate, and the homogenate then was centrifuged at $6000 \mathrm{r} / \mathrm{min}(2500 \times \mathrm{g})$ for $15 \mathrm{~min}$ at $4^{\circ}$ $\mathrm{C}$, and the supernatant was collected for the following experiments. ELISA kits and an ELISA reader (Bio-Rad Laboratories, Richmond, CA) were applied to determine the content of TNF- $\alpha$, IL-1 $\beta$, IL-6, IL-4, TGF- $\beta$ and IL-10, of which the measuring wavelength was set at $450 \mathrm{~nm}$.

\section{Luciferase Reporter Assay}

The targeting relationships involving miR-449c-5p, SNHG4 and 3' -UTR of STAT6 were verified by the luciferase reporter assay. The wild type (WT) SNHG4 sequence or the WT 3'-UTR fragment from STAT6 mRNA containing the predicted miR-449c-5p binding site was amplified and inserted into the pmirGLO dual-luciferase miRNA target expression vector (Promega, Madison, WI, USA) to establish the reporter vector pmirGLO-SNHG4-WT and pmirGLO-STAT6WT. The putative binding site of miR-449c-5p in the SNHG4 or STAT6 3' -UTR was mutated using GeneArtTM Site-Directed Mutagenesis PLUS System (cat. no. A14604; Thermo Fisher Scientific, Inc.). The mutant (Mut) SNHG4 or STAT6 3' -UTR was inserted into the pmirGLO vector to construct the reporter vector pmirGLO-SNHG4-Mut and pmirGLO-STAT6-Mut. The reporter vectors and miR-449c$5 \mathrm{p}$ mimics or NC mimics were co-transfected into HEK293 cells and the cells were incubated for $48 \mathrm{~h}$. Luciferase activity was subsequently measured using the Dual-Luciferase Reporter Assay System (Promega, Madison, WI, USA) in accordance with the protocol.

\section{Quantitative Real-Time PCR (qRT-PCR) Analysis}

Total RNA in the tissue samples or the microglia were extracted using TRIzol reagent (Invitrogen, Carlsbad, CA, USA), and miRNA and mRNA were reverse transcribed into cDNA using One Step PrimeScript miRNA cDNA synthesis kit (Invitrogen, Carlsbad, CA, USA) and PrimeScript RT kit (Invitrogen, Carlsbad, CA, USA), respectively, and then real-time fluorescence quantitative PCR was performed using (TaKaRa, Dalian, China). The expressions of SNHG4, STAT6, iNOS, CD86, Arg-1, CD206 and miR449c-5p (GAPDH and U6 were used for internal references) were evaluated using the $2^{-\Delta \Delta \mathrm{Ct}}$ method. Each experiment was repeated three times. The primers are listed in Table 1.

\section{Western Blot}

When the cells were prepared, RIPA lysis buffer (Beyotime Biotechnology, Shanghai, China) was added to isolate the total protein. Fifty microgram of total protein was separated through SDS-PAGE at $100 \mathrm{~V}$ for $2 \mathrm{~h}$ in the $12 \%$ polyacrylamide gel. It was then electrically transferred to a polyvinylidene fluoride (PVDF) membrane. After being blocked with 5\% skim milk for $1 \mathrm{~h}$ at room temperature, the membrane was rinsed 3 times with TBST for 10 min each time. Antibodies specific to STAT6 (ab32520; Abcam), p-STAT6 (ab28829; Abcam) (concentration $1: 1000$ ) were added to incubate the membrane overnight at $4{ }^{\circ} \mathrm{C}$. After the incubation, the membrane was rinsed with TBST; the membrane then was incubated with horseradish peroxidase (HRP)-labeled anti-rabbit secondary antibody (concentration 1:3000) for $1 \mathrm{~h}$ at room temperature. Subsequently, the membrane was rinsed for 3 times with TBST for $10 \mathrm{~min}$ each time. Ultimately, the imaging was performed with the application of hypersensitive ECL (Amersham Pharmacia Biotech, Little Chalfont, UK), and the grayscale of each protein was analyzed by software Image J.

\section{Statistical Analysis}

SPSS19.0 software (SPSS Inc., Chicago, IL, USA) was used to conduct the analysis. The results were expressed as mean \pm

Table I The Primer Sequences for qRT-PCR

\begin{tabular}{|c|c|c|}
\hline Genes & Primers & quences \\
\hline$m i R-449 c-5 p$ & $\begin{array}{l}\text { Forward } \\
\text { Reverse }\end{array}$ & $\begin{array}{l}\text { 5'-CAGTGTATTGCTAGCGGCTGT-3' } \\
\text { 5' -AGTGCGTGTCGTGGAGTC-3' }\end{array}$ \\
\hline SNHG4 & $\begin{array}{l}\text { Forward } \\
\text { Reverse }\end{array}$ & $\begin{array}{l}\text { 5'-GGCTAGAGTACAGTGGCTCG-3' } \\
\text { 5'-GCAAATCGCAAGGTCAGG-3' }\end{array}$ \\
\hline STAT6 & $\begin{array}{l}\text { Forward } \\
\text { Reverse }\end{array}$ & $\begin{array}{l}\text { 5'-GTCTGGTCTCCAAGATGCCC-3' } \\
\text { 5'-ATATGCTCTCAAGGGTGCTGA-3' }\end{array}$ \\
\hline iNOS & $\begin{array}{l}\text { Forward } \\
\text { Reverse }\end{array}$ & $\begin{array}{l}\text { 5'-CTGCTGGTGGTGACAAGCACATTT-3' } \\
\text { 5' -ATGTCATGAGCAAAGGCGCAGAAC-3' }\end{array}$ \\
\hline CD86 & $\begin{array}{l}\text { Forward } \\
\text { Reverse }\end{array}$ & $\begin{array}{l}\text { 5' -TCTCCACGGAAACAGCATCT-3' } \\
\text { 5' -CTTACGGAAGCACCCATGAT-3' }\end{array}$ \\
\hline Arg-I & $\begin{array}{l}\text { Forward } \\
\text { Reverse }\end{array}$ & $\begin{array}{l}\text { 5'-CACAGTCTGGCAGTTGGAAG-3' } \\
\text { 5' -GGGAGTGTTGATGTCAGTGTG-3' }\end{array}$ \\
\hline CD206 & $\begin{array}{l}\text { Forward } \\
\text { Reverse }\end{array}$ & $\begin{array}{l}\text { 5'-CAAGGAAGGTTGGCATTTGT-3' } \\
\text { 5' -CCTTTCAGTCCTTTGCAAGC-3' }\end{array}$ \\
\hline GAPDH & $\begin{array}{l}\text { Forward } \\
\text { Reverse }\end{array}$ & $\begin{array}{l}\text { 5' -GAAGGTGAGGTCGGAGTC-3' } \\
\text { 5' -GAAGAGTGGATGGGATTC-3' }\end{array}$ \\
\hline U6 & $\begin{array}{l}\text { Forward } \\
\text { Reverse }\end{array}$ & $\begin{array}{l}\text { 5' -GCTTCGGCAGCACATATACTAA-3' } \\
\text { 5' -GCTTCACAATTTGCGTGTCAT-3' }\end{array}$ \\
\hline
\end{tabular}

Abbreviations: iNOS, induced nitric oxide synthase; $C D$, cluster of differentiation; GAPDH, glyceraldehyde-3-phosphate dehydrogenase. 
standard deviation $(x \pm s)$. Kolmogorov-Smirnov test was used to examine whether the data were normally distributed or not. For normally distributed data, unpaired $t$ test was used to make a comparison between 3 groups. One-way ANOVA analysis was used to make a comparison among 3 or more groups, and after that, Newman-Keuls analysis was used to make a comparison between 2 groups. If the data were not normally distributed, nonparametric Mann-Whitney test was used to make a comparison between 2 groups. Kruskal-Wallis test was used to make comparison among 3 or more groups, and after that Mann-Whitney test was further performed to make comparison between 2 groups. Each experiment was repeated 3 times with a significant difference of $P<0.05$.

\section{Results}

\section{SNHG4 Expression in Cerebral Infarction Patients and Rat MCAO Model}

To investigate the expression of SNHG4 in blood and cerebrospinal fluid derived from patients with ACI, we collected samples from 30 patients and 30 healthy controls. qRT-PCR results demonstrated that the expression of SNHG4 in the samples, both blood and cerebrospinal fluid, collected from patients with ACI, were remarkably down-regulated in comparison with the normal group (Figure 1A-B). Additionally, in the rat models of cerebral ischemia reperfusion, the expression of SNHG4 in the brain tissues of MCAO group was significantly down-regulated (vs sham group) (Figure 1C). Immunohistochemical staining and LDH release assay showed that the number of caspase-3-positive cells and LDH release were markedly increased in the MCAO group (vs sham group) (Figure 1D-E). The results of ELISA showed that in MCAO group, the expressions of pro-inflammatory cytokines IL-1 $\beta$, IL- 6 and TNF- $\alpha$ were remarkably higher than those of sham group, while the expressions of antiinflammatory cytokines IL-4, IL-10 and TGF- $\beta$ were dramatically decreased (Figure 1F). These data suggested that the down-regulation of SNHG4 could probably contribute to the process of cerebral ischemia-reperfusion injury.

\section{SNHG4 Overexpression Inhibited Microglia Activation and Inflammatory Response}

To study the association between SNHG4 and microglia activation, the microglia (HAPI cells) were induced by LPS in vitro. The expressions of M1 and M2 polarization markers in the microglia were detected by qRT-PCR. The data revealed that the expressions of M1 markers iNOS and CD86 increased significantly under the induction of
LPS, while the expressions of M2 markers Arg1 and CD206 decreased notably (vs control group) (Figure 2A). The expression of SNHG4 in the microglia treated with LPS was remarkably lower than that of the control (Figure 2B). Furthermore, the overexpression and the knock-down of SNHG4 microglia cell lines were established, respectively, and the OGD models were constructed (Figure 2C). It was implied that OGD facilitated the overexpressions of M1 markers iNOS and CD86, but inhibited the expressions of M2 markers Arg1 and CD206; additionally, SNHG4 knockdown exacerbated these phenomena, while SNHG4 overexpression functioned oppositely (Figure 2D). Besides, the effect of SNHG4 on inflammatory response was further detected by ELISA kit. The results implied that, in the cell culture supernatant, the overexpression of SNHG4 could inhibit the releases of proinflammatory factors induced by OGD and promoted the expressions of anti-inflammatory factors, while SNHG4 knockdown functioned oppositely (Figure 2E). These results indicated that SNHG4 could inhibit the activation of microglia and ameliorate the inflammatory response caused by OGD.

\section{SNHG4 Inhibited the Expression of miR-449c-5p by Targeting It}

To probe how SNHG4 regulated the activation of microglia, the binding site where SNHG4 binds with miR-449c$5 \mathrm{p}$ was predicted by the online website StarBase (Figure $3 \mathrm{~A}$ ), and the targeting relationship between SNHG4 and miR-449c-5p was further verified by luciferase assay. The results revealed that miR-449c-5p remarkably inhibited the luciferase activity of SNHG4-WT, but it could not inhibit the luciferase activity of SNHG4-Mut (Figure 3B). Furthermore, qRT-PCR was employed to detect miR$449 c-5 p$ expression. The data showed that miR-449c-5p expression was significantly down-regulated in HAPI cells with SNHG4 overexpression and significantly upregulated in HAPI cells with SNHG4 knocked down (Figure 3C). The results indicated that miR-449c-5p was the target of SNHG4 and its expression was negatively regulated by SNHG4. Additionally, miR-449c-5p expression was markedly up-regulated in the blood and cerebrospinal fluid samples derived from the patients with ACI (vs Control group) (Figure 3D and E). Consistently, in the MCAO rat brain tissues and LPS-induced microglia, miR-449c-5p expression was remarkably up-regulated compared to the control groups (Figure $3 \mathrm{~F}$ and $\mathrm{G}$ ). These 

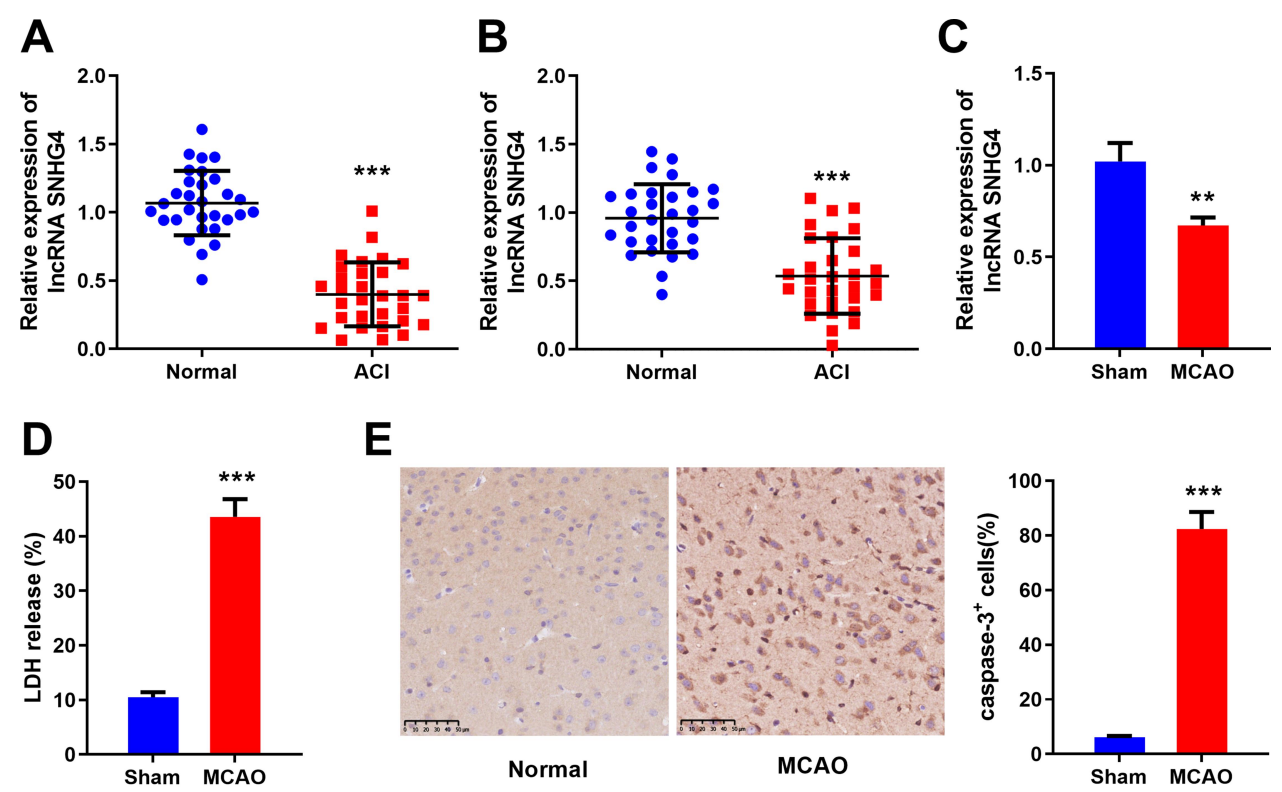

E
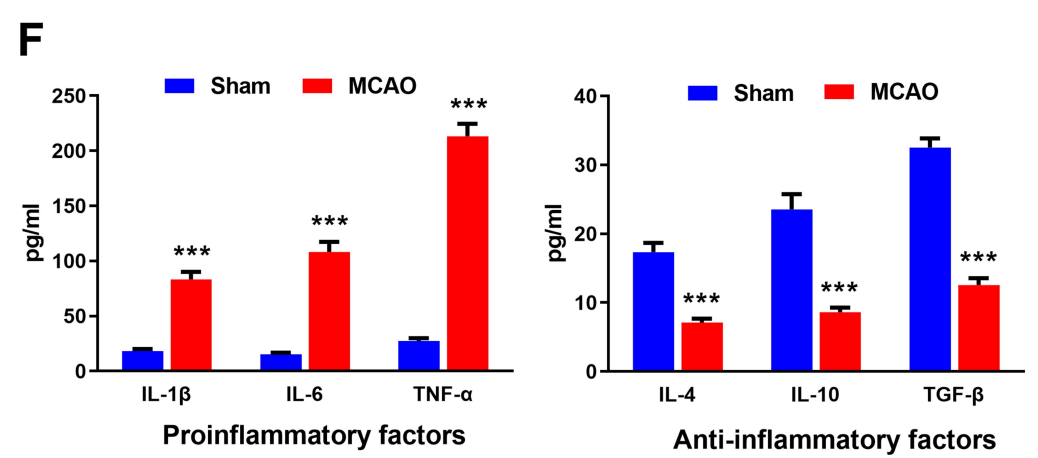

Figure I Expression of SNHG4 in cerebral infarction patients and rat MCAO model. (A-C) qRT-PCR was used to detect the expression of SNHG4 in blood and cerebrospinal fluid samples of patients and the brain tissues of MCAO animal model; (D) LDH detection assay was used to detect the expression of LDH in infarcted tissue of MCAO rats. (E) The activity of caspase- 3 in the brain tissue of MCAO rats was detected by immunohistochemical staining; (F) ELISA was used to detect the levels of proinflammatory factors (IL-I $\beta$, IL-6, TNF- $\alpha$ ) and anti-inflammatory factors (IL-4, IL- I0, TGF- $\beta$ ) in the brain tissue of MCAO rats. **P< 0.0 I, and $* * * P<0.00$ I versus the healthy controls or sham group.

Abbreviations: ACl, acute cerebral infarction; MCAO, middle cerebral artery occlusion; LDH, lactate dehydrogenase; ELISA, enzyme-linked immunosorbent assay; IL, interleukin; TNF, tumor necrosis factor; TGF, transforming growth factor.

data suggested miR-449c-5p was an injurious factor during cerebral ischemia-reperfusion injury.

\section{The Role of miR-449c-5p}

\section{Over-Expression and Inhibition on}

\section{Activation and Inflammation of Microglia}

To explore whether SNHG4 regulates microglial activation by modulating the expression of miR-449c-5p, we constructed miR-449c-5p over-expression and inhibition cell lines, respectively (Figure 4A). qRT-PCR was employed to detect the expressions of M1 and M2 markers in the microglia. The data implied that the M1 marker was remarkably decreased in the miR-449c-5p inhibition cell line, while the M2 marker was significantly up-regulated (vs NC inhibitors+OGD group).
(Figure 4B). The results of the ELISA kit revealed that the expressions of pro-inflammatory factors were notably decreased in the miR-449c-5p inhibition cell line, while the expressions of anti-inflammatory factors were significantly increased (vs NC inhibitors+OGD group) (Figure $4 C)$. Conversely, miR-449c-5p mimics exerted opposite effects (Figure 4B and C). The data implied that miR$449 \mathrm{c}-5 \mathrm{p}$ regulated the process in which OGD activated the microglia and induced inflammatory response.

\section{miR-449c-5p Binds with the 3'UTR of STAT6}

The online target gene prediction database TargetScan was employed to predict the target gene of miR-449c-5p, and we found that miR-449c-5p could probably bind to the specific regions of STAT6 3'UTR (Figure 5A). We further validated 

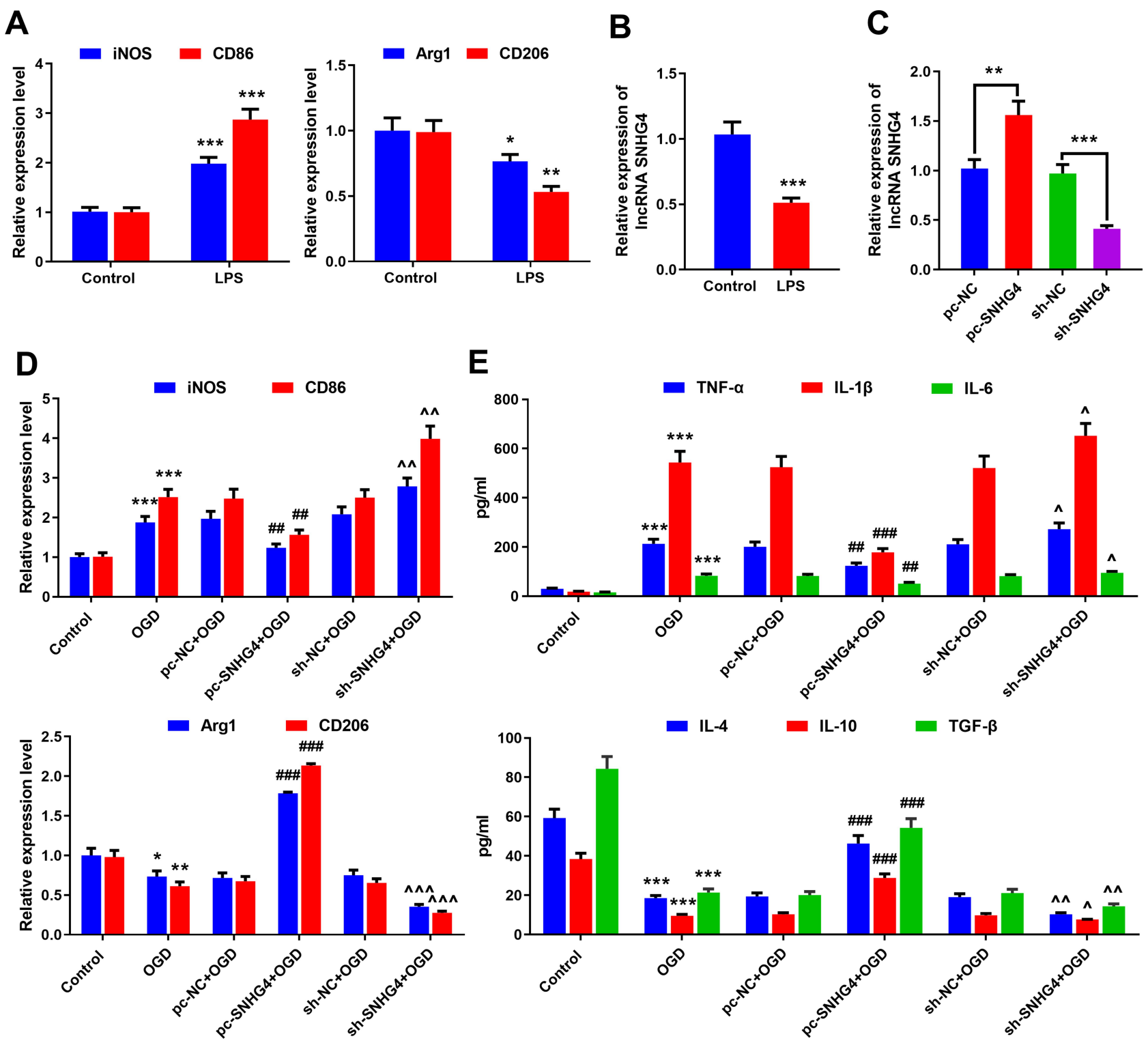

$\mathbf{E}$
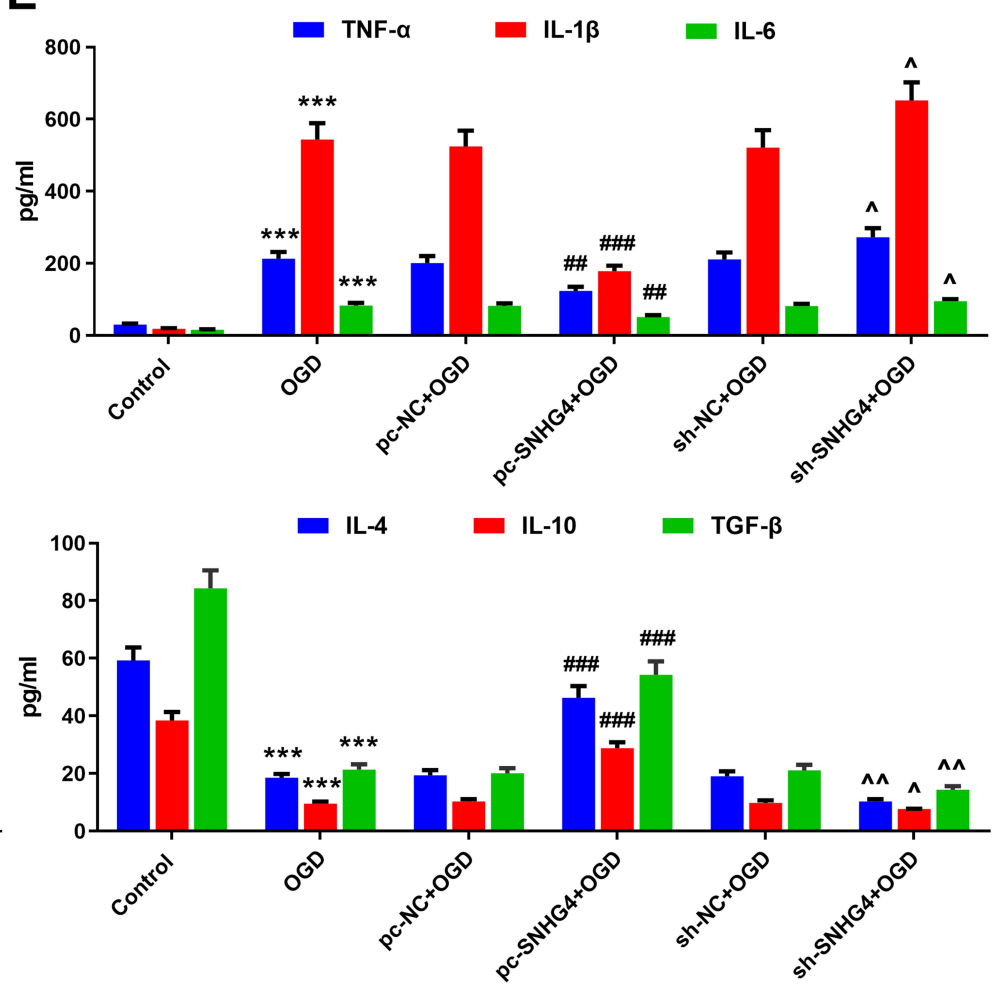

Figure 2 SNHG4 overexpression inhibits microglial activation and inflammatory responses. (A) qRT-PCR was used to detect the expression of MI markers iNOS and CD86 and M2 markers ArgI and CD206 in microglia treated with LPS; (B) qRT-PCR was used to detect the expression of SNHG4 in microglia treated with LPS; (C) qRT-PCR was used to detect the expression of SNHG4 cell models with SNHG4 overexpression and knockdown; (D) qRT-PCR was used to detect the expression of MI markers iNOS and CD86 and M2 ArgI and CD206 in microglia treated with OGD. (E) ELISA was used to detect the levels of pro-inflammatory cytokines (IL-I $\beta$, IL-6, TNF- $\alpha$ ) and antiinflammatory factors (IL-4, IL-I0, TGF- $\beta$ ). $* P<0.05$, $* * P<0.01$, and $* * * P<0.001$ versus the control group; \#\#P< 0.01 and \#\#P< 0.00 I versus the $P C-N C+O G D$ group; $\wedge P<$ $0.05, \wedge \wedge P<0.01$ and $\wedge \wedge \wedge P<0.001$ versus the sh-NC+OGD group.

Abbreviations: iNOS, induced nitric oxide synthase; CD, cluster of differentiation; LPS, lipopolysaccharide; OGD, oxygen-glucose deprivation; Pc, pc-DNA3.I; NC, negative control; sh, short-hairpin RNA; TNF, tumor necrosis factor; IL, interleukin; TGF, transforming growth factor.

the targeting relationship between them by dual-luciferase reporter assay. The results implied that miR-449c-5p could reduce the luciferase activity of STAT6-WT, but had no effect on STAT6-Mut (Figure 5B). The qRT-PCR results revealed that miR-449c-5p remarkably inhibited STAT6 expression (vs NC mimics group) (Figure 5C). Additionally, pcSNHG4 plasmid was transfected into the microglial cell line treated with miR-449c-5p mimics. The qRT-PCR experiment demonstrated that SNHG4 over-expression remarkably suppressed the expression of miR-449c-5p, while STAT6 was remarkably up-regulated (vs miR-449c-5p mimics + pcNC group) (Figure 5D-E).

The Roles of SNHG4/miR-449c-5p Axis in Activation and Inflammatory Response of Microglia

To further probe the effect of SNHG4/miR-449c-5p/ STAT6 axis on microglial inflammatory response, 

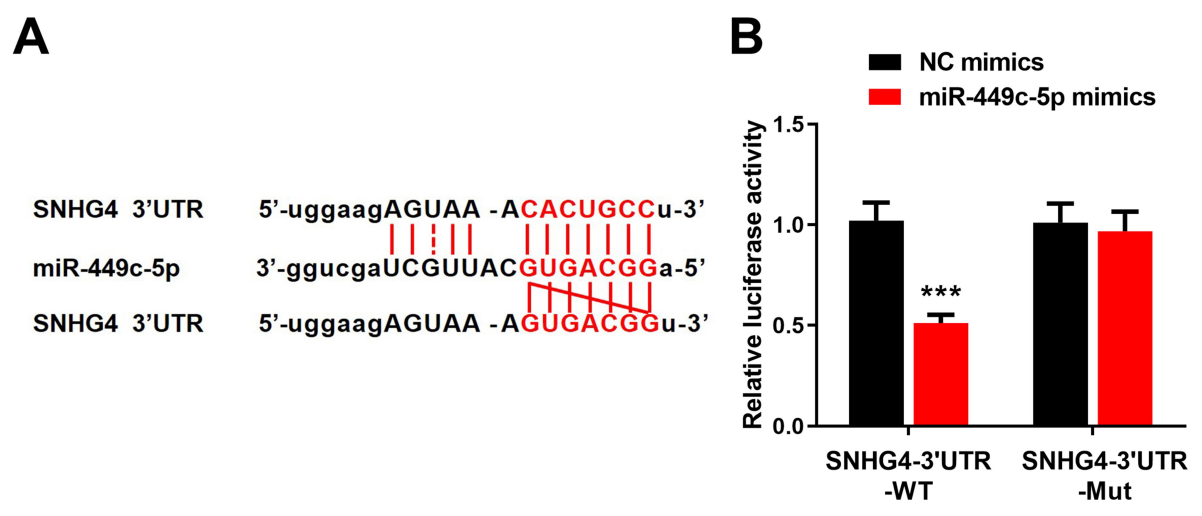

C
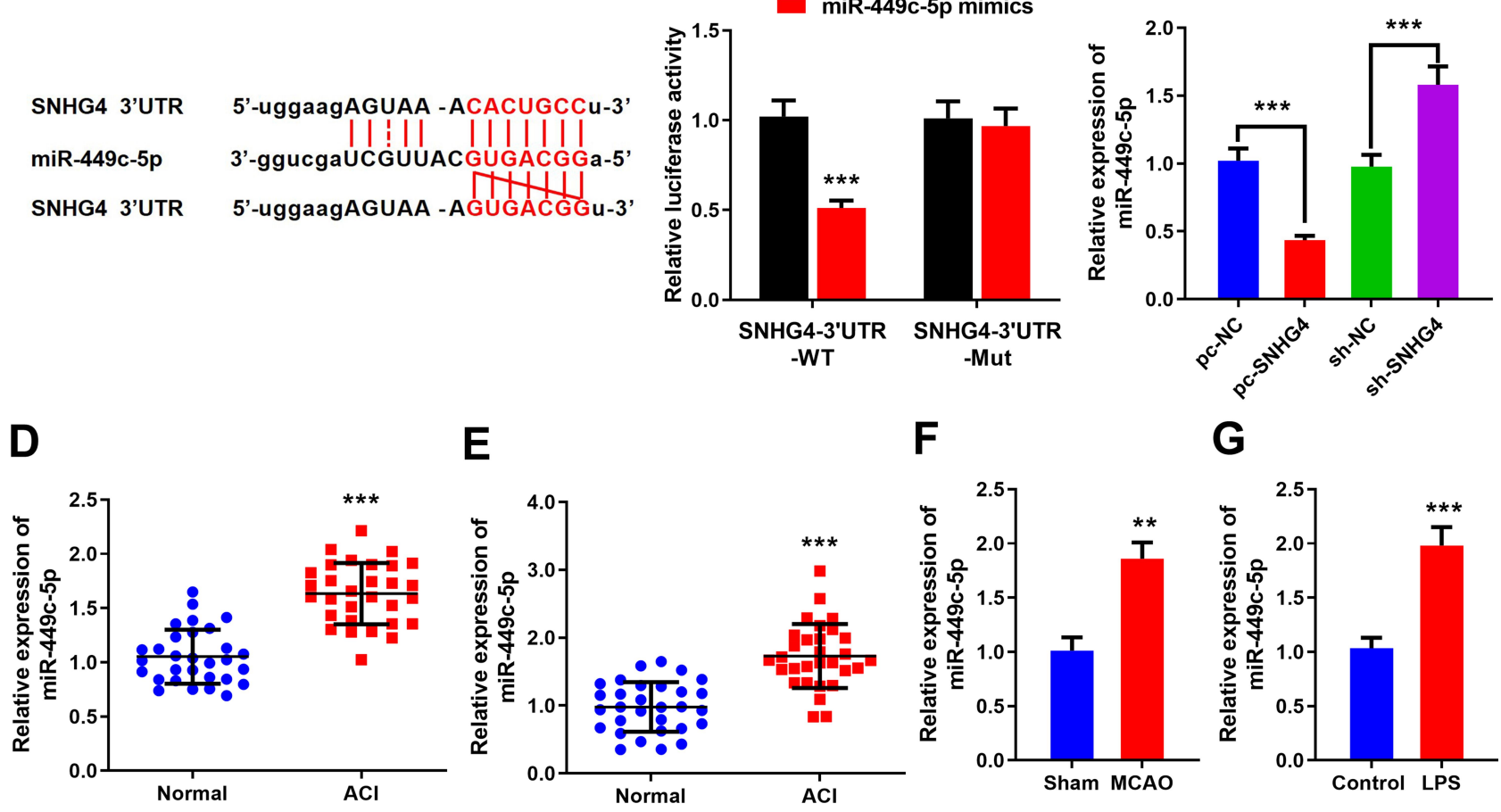

G

Figure 3 SNHG4 inhibits miR-449c-5p by targeting it. (A) The sequence of binding site to miR-449c-5p in the of SNHG4; (B) Luciferase activity assay was used to verify that SNHG4 could absorb miR-449c-5p; (C) qRT-PCR was used to detect the expression of miR-449c-5p in SNHG4 overexpression and low expression cell lines; (D-F) qRTPCR was used to detect the expression of miR-449c-5p in blood, cerebrospinal fluid of patients and brain tissue of MCAO rats; (G) the expression of miR-449c-5p in microglia was detected by $\mathrm{qRT}$-PCR after LPS treatment. ** $P<0.01$ and $* * * P<0.001$.

Abbreviations: NC, negative control; WT, wild type; Mut, mutant; pc, pc-DNA3.I; sh, short-hairpin RNA; ACI, acute cerebral infarction; MCAO, middle cerebral artery occlusion; LPS, lipopolysaccharide.

qRT-PCR was adopted to detect M1 and M2 markers' expressions in the microglia treated with OGD. Meanwhile, we used the ELISA to detect the level of inflammatory cytokines. The data revealed that miR$449 c-5 p$ mimics significantly increased the expression of M1 markers and pro-inflammatory factors, inhibited the expression of M2 markers and anti-inflammatory factors, promoting the activation of microglia. But SNHG4 significantly reversed these effects (Figure 6A-B). Moreover, we used the medium (without microglial cells) in which different microglial cell lines treated with OGD, to culture hippocampal neuronal cells PC-12. Then, the viability and LDH release rate of hippocampal neuronal cells were analyzed with CCK-8 assay and LDH detection assay, respectively. The results suggested that the viability of the hippocampal neuronal cells maintained in miR-449c$5 p$ mimics + pc-SNHG4 cell line medium was significantly higher than that in miR-449c-5p mimics + pc-NC cell line medium, while the release rate of LDH had the reverse trend (Figure 6C-D). It suggested that SNHG4 could inhibit miR-449c-5p expression, weaken the activation and inflammatory responses of microglia induced by OGD, and ameliorate the injury of hippocampal neurons induced by OGD. Additionally, Western blot assay showed that the overexpression of miR-449c-5p reduced the expression level and phosphorylation level of STAT6 in microglia induced by OGD, while the overexpression of SNHG4 promoted the expression of phosphorylation of STAT6 and STAT6 (Figure 7). Therefore, we concluded that SNHG4 could activate STAT6 signaling pathway by inhibiting the expression of miR-449c-5p, so as to ameliorate the inflammatory response and neuronal damage caused by microglia.

\section{Discussion}

Ischemic stroke is a common cause of the impairment in the CNS. In recent years, ncRNAs have become the focus of research on ischemic stroke. LncRNAs can modulate transcription and translation, and miRNAs directly target mRNAs to modulate post-transcriptional gene expression. ${ }^{19,20}$ However, the role of ncRNA in stroke still remains further investigation. 
A

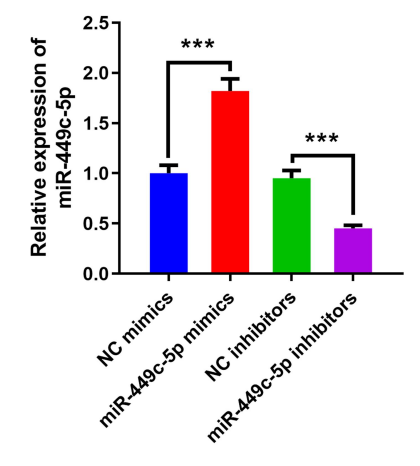

B

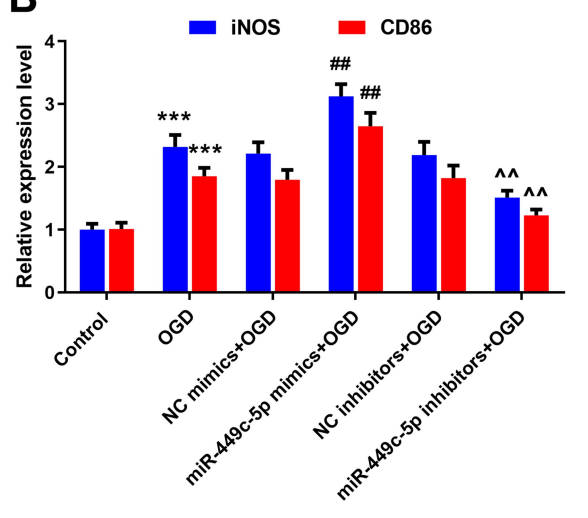

C

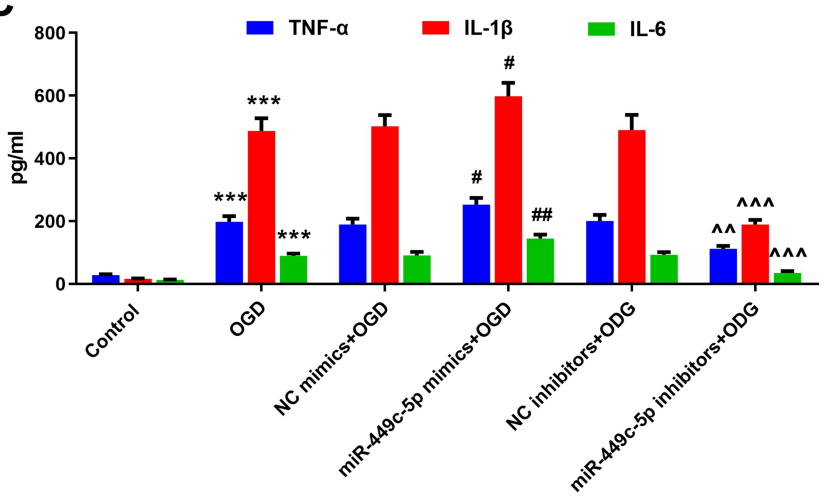

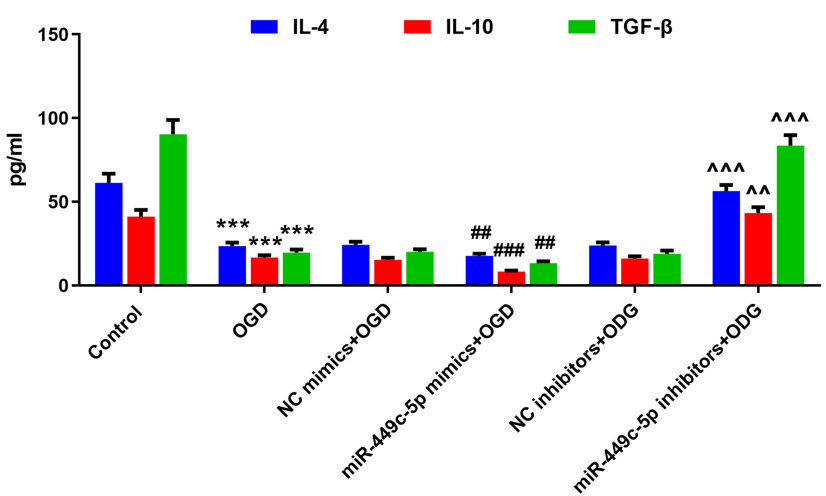

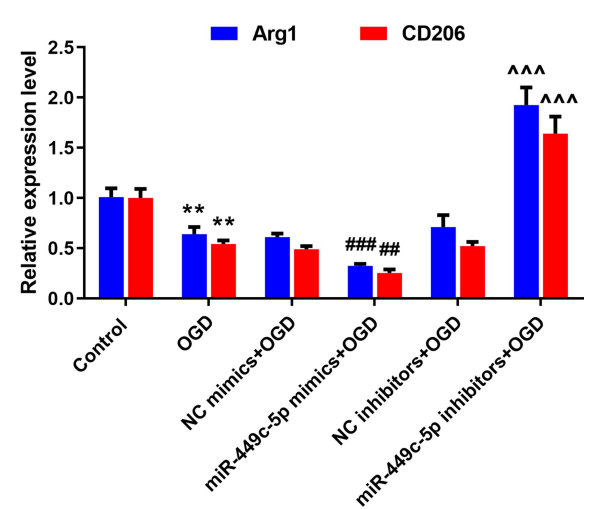

Figure 4 Effects of overexpression and inhibition of miR-449c-5p on activation and inflammatory response of microglia. (A) The overexpression and inhibition of miR-449c$5 p$ cell models were constructed; (B) qRT-PCR was used to detect the expression of MI markers iNOS and CD86 and M2 markers ArgI and CD206 in microglia treated with OGD; (C) ELISA was used to detect the levels of pro-inflammatory factors (IL-I $\beta$, IL-6, TNF- $\alpha$ ) and anti-inflammatory factors (IL-4, IL-I0, TGF- $\beta$ ). $* * P<0.0$ I and $* * * P<0.00$ I versus the control group; $\# P<0.05, \# P<0.01$ and \#\#P<0.00I versus the NC mimics + OGD group; $\wedge \wedge P<0.01$ and $\wedge \wedge \wedge P<0.001$ versus the NC inhibitors $+O G D$ group.

Abbreviations: NC, negative control; iNOS, induced nitric oxide synthase; CD, cluster of differentiation; OGD, oxygen-glucose deprivation; TNF, tumor necrosis factor; IL, interleukin; TGF, transforming growth factor.

Microglia, belonging to the mononuclear phagocytic family, are indispensable immune cells in the CNS and implicated in various neurological diseases, such as ischemic stroke, HIV encephalopathy, Parkinson's disease, and Alzheimer's disease. ${ }^{21}$ The neuroinflammation mediated by microglia plays a key role in the injury of CNS and the progression of the CNS-related diseases. ${ }^{22}$ After stroke, the activation of stroke-induced microglia results in the release of a variety of inflammatory mediators, many of which have cytotoxicity or cytoprotective effects. Alternately activated microglia (M2) promote tissue repair via secreting anti-inflammatory mediators and growth factors; ${ }^{23,24}$ conversely, regularly activated microglia (M1) can secrete a variety of inflammatory and neurotoxic compounds, such as nitric oxide, TNF- $\alpha$, IL- 6 and reactive oxygen species (ROS), which can cause damage to neurons. ${ }^{10}$ Based on these findings, it is increasingly commonly acknowledged that the polarization of microglia has dual effect on neuronal apoptosis, neurogenesis, and the recovery of brain function after cerebral ischemia. M1 is characterized by the induction of STAT1 and NF- $\kappa \mathrm{B}$ transcription factors, as well as the production of pro-inflammatory cytokines including TNF- $\alpha$, IL-1, IL-6 and IL-12. ${ }^{25,26}$ While M2 is characterized by the activation of the transcription factor STAT6, increased expression of the mannose receptor CD206, and the production of cytokines such as TGF- $\beta$, CCL18, and IL$1 \mathrm{Ra}^{27} \mathrm{In}$ our research, the microglia were activated after MCAO and secreted IL1 $\beta$, TNF- $\alpha$, and IL6. Meanwhile, M1 markers iNOS and CD86 were up-regulated. It was consistent with the results occurred on microglia treated with OGD.

Over these years, IncRNA has been increasingly studied in the development of cerebral ischemic stroke. For example, lncRNA-1810034E14Rik helps protect the brain from the damage caused by cerebral ischemia and inhibits 


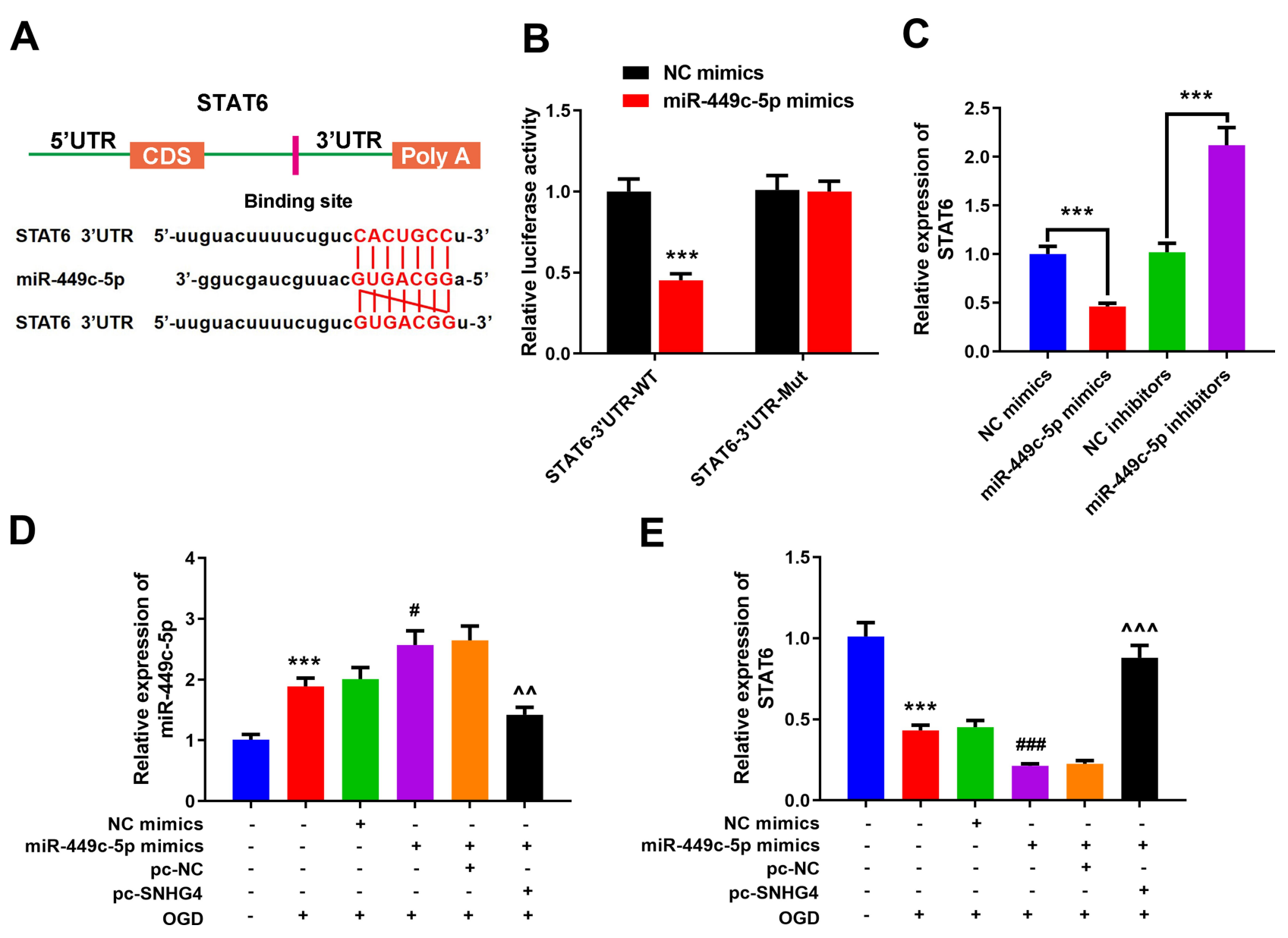

Figure 5 miR-449c-5p can bind to STAT6 by targeting it. (A) Prediction of targeted binding sites of miR-449c-5p and the 3'UTR of STAT6; (B) Luciferase activity assay was used to verify that miR-449c-5p could target the 3'UTR of STAT6; (C) qRT-PCR was used to detect the expression of STAT6 in miR-449c-5p overexpression and low expression cell lines; (D, E) qRT-PCR was used to detect the expression of miR-449c-5p and STAT6 after the co-transfection. *** $P<0.00$ I versus the control group; \#P< 0.05 and 0.001 versus the NC mimics+OGD group; $\wedge \wedge P<0.01$ and $\wedge \wedge \wedge P<0.001$ versus the miR-449c-5p mimics $+p c-N C+O G D$ group.

Abbreviations: NC, negative control; WT, wild type; Mut, mutant; pc, pc-DNA3.I; OGD, oxygen-glucose deprivation.

NF- $\kappa \mathrm{B}$ pathway to attenuate neuroinflammation. ${ }^{14}$ It is reported that lncRNA H19 can promote neuroinflammation by driving HDAC1-dependent M1 microglial polarization. ${ }^{28}$ In this study, the data revealed that SNHG4 expression was significantly down-regulated in patients with ACI, MCAO rat models as well as LPS and OGD-treated microglia. The over-expression of SNHG4 reversed the increase of pro-inflammatory cytokines IL-1 $\beta$, IL- 6 and TNF- $\alpha$ in MCAO rat models, LPS and OGDtreated microglia, and promoted the release of antiinflammatory factors IL-4, IL-10 and TGF- $\beta$. And the expressions of M2 type microglia markers Arg-1 and CD206 were remarkably up-regulated. In the previous studies, SNHG4 is mainly linked with disease progression of multiple cancers including osteosarcoma, lung cancer, cervical cancer and prostate cancer. ${ }^{29-32}$ To our best knowledge, there are no reports on its role in nervous system diseases and inflammatory regulation. For the first time, our work proved SNHG4 was a crucial regulator during cerebral ischemia-reperfusion injury via modulating neuroinflammation.

miRNAs are critical for regulating the differentiation of macrophages into regularly and alternately activated macrophages. ${ }^{33}$ For instance, in microglia, miR-124 downregulates the levels of M1 markers IL-6, TNF- $\alpha$, iNOS and up-regulates the expressions of M2 markers TGF- $\beta 1$, Arg 1 and FIZZ1. ${ }^{34}$ miR-449c-5p is a rarely investigated miRNA, and limited studies suggest that it is associated with osteogenic differentiation of valve interstitial cells and the progression of hepatocellular carcinoma. ${ }^{35,36} \mathrm{In}$ this work, for the first time, we proved that miR-449c-5p participated in the activation of microglia and inflammatory response in cerebral ischemia. Additionally, through bioinformatics prediction and luciferase reporter assay, we 
A

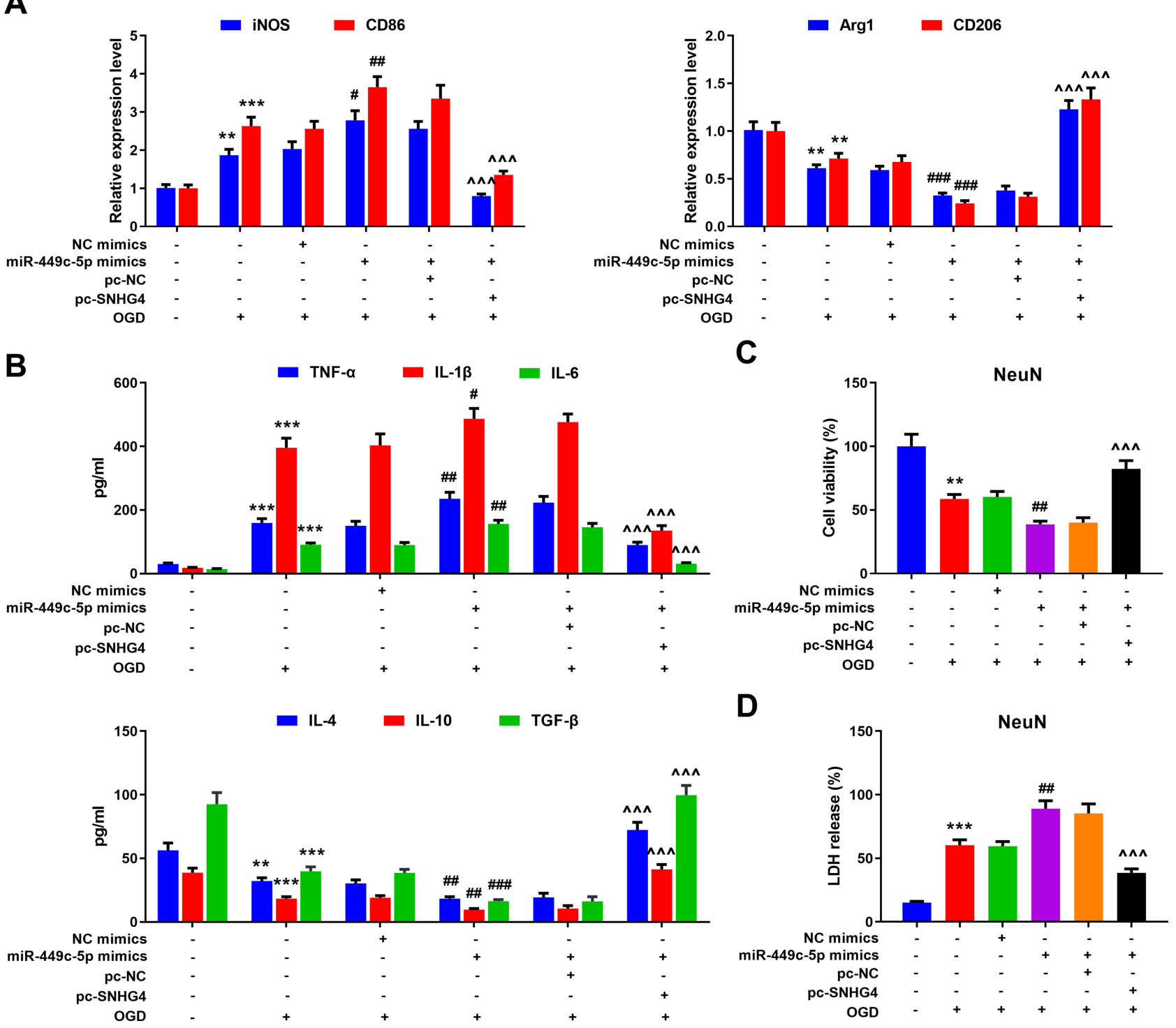

Figure 6 Overexpression of SNHG4 reduced activation and inflammatory response of microglia via regulating miR-449c-5p. (A) qRT-PCR was employed to detect the expression of MI markers iNOS and CD86 and M2 markers ArgI and CD206 mRNA in microglia treated with OGD; (B) The changes of pro-inflammatory cytokines (IL-I $\beta$, IL-6, TNF- $\alpha$ ) and anti-inflammatory cytokines (IL-4, IL-I0, TGF- $\beta$ ) were detected by ELISA; (C) The effect of microglia culture medium on the activity of hippocampal neurons was detected by CCK-8 assay; (D) The release of LDH from hippocampal neurons was detected. $* * P<0.01$ and $* * * P<0.001$ versus the control group; \#P< 0.05 ,

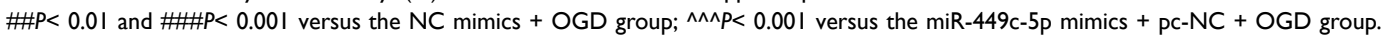

Abbreviations: iNOS, induced nitric oxide synthase; CD, cluster of differentiation; NC, negative control; pc, pc-DNA3.I; OGD, oxygen-glucose deprivation; TNF, tumor necrosis factor; IL, interleukin; NeuN, neuron; TGF, transforming growth factor; LDH, lactate dehydrogenase.

validated that SNHG4 could specifically inhibit the expression of miR-449c-5p, and facilitate microglia's polarization towards M2 and inhibited the inflammatory response of microglia.

STAT signaling pathway is widely implicated in cell proliferation, differentiation, apoptosis and immune regulation, and functions critically in the signal transduction of a variety of cytokines. ${ }^{37}$ STAT6 functions as cytoplasmic transcription factor modulated via the phosphorylation and can be activated in response to IL-4 and IL-13. ${ }^{38}$ With the phosphorylation of the key tyrosine residue, STAT6 becomes dimerized, transfers from the cytoplasm to the nucleus, resulting in the up-regulation of specific transcription of the target gene. ${ }^{38}$ Studies have shown that IL4-STAT6 signaling induces macrophages to desensitize endogenous signals related to microbes, stress and injury through direct transcriptional inhibition of inflammatory enhancers. ${ }^{39}$ It has been found that hyperglycemia promotes M1 polarization of intrahepatic macrophages obtained from liver exposed to APAP and meanwhile 

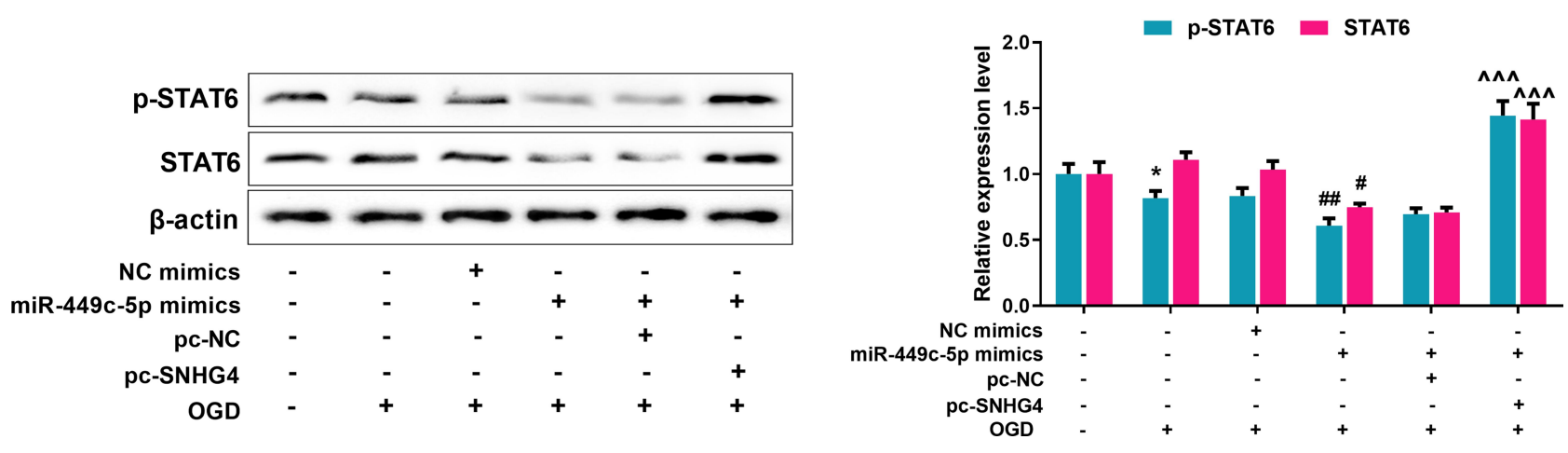

Figure 7 SNHG4 promotes the activation of STAT6 signal pathway via regulating miR-449c-5p. Western blot was used to detect the expression and relative expression of STAT6 total protein and phosphorylated protein. ${ }^{*} P<0.05$ versus the $N C$ mimics group; $\# P<0.05$ and \#\#P 0.01 versus the NC mimics $+O G D$ group; $\wedge \wedge \wedge P<0.00$ I versus the miR-449c-5p mimics + pc-NC + OGD group.

Abbreviations: STAT, signal transducer and activator of transcription 6; p-STAT6, phospho-STAT6; NC, negative control; pc, pc-DNA3.I; OGD, oxygen-glucose deprivation.

inhibits M2 polarization, accompanied by the increased induction of MCP-1 and iNOS, decreased induction of Arg-1 and CD206, increased activation of STAT1 and decreased activation of STAT6. ${ }^{40}$ In this work, we found that miR-449c-5p could target STAT6 to inhibit its expression. As expected, over-expression of SNHG4 could significantly promote the phosphorylation of STAT6, upregulate the expression of Arg-1 and CD206, and facilitate the transformation of microglia treated with ODG from M1 to M2. Meanwhile, the expression of antiinflammatory factors IL-4, IL-10 and TGF- $\beta$ were remarkably increased, which greatly reduced the toxic effect of inflammation on neurons.

In summary, our work validates that the down-regulation of SNHG4 is involved in cerebral ischemia-reperfusion injury. Up-regulation of SNHG4 inhibits the expression of miR-449c-5p and activates the STAT6 signaling pathway, thereby inhibiting the M1 polarization of microglia and reducing inflammation, suggesting restoration of SNHG4 is a promising strategy in the treatment of ischemic stroke.

\section{Data Sharing Statement}

The data used to support the findings of this study are available from the corresponding author upon request.

\section{Ethics Statement}

Our study was approved by the Ethics Review Board of the First Affiliated Hospital of China Medical University.

\section{Author Contributions}

All authors made substantial contributions to conception and design, acquisition of data, or analysis and interpretation of data; took part in drafting the article or revising it critically for important intellectual content; gave final approval of the version to be published; and agree to be accountable for all aspects of the work.

\section{Funding}

There is no funding to report.

\section{Disclosure}

The authors declare that they have no competing interests.

\section{References}

1. Lundberg GP, Volgman AS. Burden of stroke in women. Trends Cardiovasc Med. 2016;26(1):81-88. doi:10.1016/j.tcm.2015.04.010

2. Couchie D, Vaisman B, Abderrazak A. Correction to: heart disease and stroke statistics-2017 update: a report from the american heart association. Circulation. 2017;136(10):e196. doi:10.1161/ CIRCULATIONAHA.117.027612

3. Fisher M, Saver JL. Future directions of acute ischaemic stroke therapy. Lancet Neurol. 2015;14(7):758-767. doi:10.1016/S14744422(15)00054-X

4. Moskowitz MA, Lo EH, Iadecola C. The science of stroke: mechanisms in search of treatments. Neuron. 2010;67(2):181-198. doi:10.1016/j.neuron.2010.07.002

5. Hankey GJ. Stroke. Lancet (London, England). 2017;389 (10069):641-654. doi:10.1016/S0140-6736(16)30962-X

6. Jin R, Yang G, Li G. Inflammatory mechanisms in ischemic stroke: role of inflammatory cells. J Leukoc Biol. 2010;87(5):779-789. doi:10.1189/jlb.1109766

7. Tremblay ME, Stevens B, Sierra A, Wake H, Bessis A, Nimmerjahn A. The role of microglia in the healthy brain. J Neurosci. 2011;31 (45):16064-16069. doi:10.1523/JNEUROSCI.4158-11.2011

8. Kettenmann H, Hanisch UK, Noda M, Verkhratsky A. Physiology of microglia. Physiol Rev. 2011;91(2):461-553. doi:10.1152/physrev. 00011.2010

9. Neher JJ, Emmrich JV, Fricker M, Mander PK, Thery C, Brown GC. Phagocytosis executes delayed neuronal death after focal brain ischemia. Proc Natl Acad Sci U S A. 2013;110(43):E4098-107. doi:10.1073/pnas.1308679110

10. Lucas SM, Rothwell NJ, Gibson RM. The role of inflammation in CNS injury and disease. Br J Pharmacol. 2006;147(1):S232-40. doi:10.1038/sj.bjp. 0706400 
11. Boutin H, LeFeuvre RA, Horai R, Asano M, Iwakura Y, Rothwell NJ Role of IL-1alpha and IL-1beta in ischemic brain damage. $J$ Neurosci. 2001;21(15):5528-5534. doi:10.1523/JNEUROSCI.2115-05528.2001

12. Wang Y, Pan WY, Ge JS, et al. A review of the relationship between long noncoding RNA and post-stroke injury repair. J Int Med Res. 2019;300060519867493.

13. Mehta SL, Kim T, Vemuganti R. Long noncoding RNA FosDT promotes ischemic brain injury by interacting with REST-associated chromatin-modifying proteins. $J$ Neurosci. 2015;35(50):164 43-16449. doi:10.1523/JNEUROSCI.2943-15.2015

14. Zhang X, Zhu XL, Ji BY, et al. LncRNA-1810034E14Rik reduces microglia activation in experimental ischemic stroke. J Neuroinflammation. 2019;16(1):75. doi:10.1186/s12974-019-1464-x

15. Liu B, Li J, Cairns MJ. Identifying miRNAs, targets and functions. Brief Bioinformatics. 2014;15(1):1-19. doi:10.1093/bib/bbs075

16. Ouyang YB, Stary CM, Yang GY, Giffard R. microRNAs: innovative targets for cerebral ischemia and stroke. Curr Drug Targets. 2013;14 (1):90-101. doi:10.2174/138945013804806424

17. Yin KJ, Deng Z, Huang H, et al. miR-497 regulates neuronal death in mouse brain after transient focal cerebral ischemia. Neurobiol Dis. 2010;38(1):17-26. doi:10.1016/j.nbd.2009.12.021

18. Huang L, Ma Q, Li Y, Li B, Zhang L. Inhibition of microRNA-210 suppresses pro-inflammatory response and reduces acute brain injury of ischemic stroke in mice. Exp Neurol. 2018;300:41-50. doi:10.1016/j.expneurol.2017.10.024

19. Saugstad JA. Non-coding RNAs in stroke and neuroprotection. Front Neurol. 2015;6:50. doi:10.3389/fneur.2015.00050

20. Abraham JM, Meltzer SJ. Long noncoding RNAs in the pathogenesis of barrett's esophagus and esophageal carcinoma. Gastroenterology. 2017;153(1):27-34. doi:10.1053/j.gastro.2017.04.046

21. Salter MW, Beggs S. Sublime microglia: expanding roles for the guardians of the CNS. Cell. 2014;158(1):15-24. doi:10.1016/j. cell.2014.06.008

22. Chen S, Dong Z, Cheng M, et al. Homocysteine exaggerates microglia activation and neuroinflammation through microglia localized STAT3 overactivation following ischemic stroke. $J$ Neuroinflammation. 2017;14(1):187. doi:10.1186/s12974-017-0963-x

23. Patel AR, Ritzel R, McCullough LD, Liu F. Microglia and ischemic stroke: a double-edged sword. Int J Physiol Pathophysiol Pharmacol. 2013;5(2):73-90.

24. Shin JA, Lim SM, Jeong SI, Kang JL, Park EM. Noggin improves ischemic brain tissue repair and promotes alternative activation of microglia in mice. Brain Behav Immun. 2014;40:143-154 doi:10.1016/j.bbi.2014.03.013

25. Ma Y, Wang J, Wang Y, Yang GY. The biphasic function of microglia in ischemic stroke. Prog Neurobiol. 2017;157:247-272. doi:10.1016/ j.pneurobio.2016.01.005

26. Van Ginderachter JA, Movahedi K, Hassanzadeh Ghassabeh G, et al. Classical and alternative activation of mononuclear phagocytes: picking the best of both worlds for tumor promotion. Immunobiology. 2006;211(6-8):487-501. doi:10.1016/j.imbio.2006.06.002

27. Gordon S, Martinez FO. Alternative activation of macrophages: mechanism and functions. Immunity. 2010;32(5):593-604. doi:10.10 16/j.immuni.2010.05.007
28. Wang J, Zhao H, Fan Z, et al. Long Noncoding RNA H19 promotes neuroinflammation in ischemic stroke by driving histone Deacetylase 1-dependent M1 microglial polarization. Stroke. 2017;48 (8):2211-2221. doi:10.1161/STROKEAHA.117.017387

29. $\mathrm{Xu} \mathrm{R}$, Feng F, Yu X, Lao ZL. LncRNA SNHG4 promotes tumour growth by sponging miR-224-3p and predicts poor survival and recurrence in human osteosarcoma. Cell Prolif. 2018;51(6):e12515. doi:10.1111/cpr.12515

30. Tang Y, Wu L, Zhao M, et al. LncRNA SNHG4 promotes the proliferation, migration, invasiveness, and epithelial-mesenchymal transition of lung cancer cells by regulating miR-98-5p. Biochem Cell Biol. 2019;97(6):767-776. doi:10.1139/bcb-2019-0065

31. Li H, Hong J, Wijayakulathilaka W. Long non-coding RNA SNHG4 promotes cervical cancer progression through regulating c-Met via targeting miR-148a-3p. Cell Cycle. 2019;18(23):3313-3324. doi:10.1080/15384101.2019.1674071

32. Duan WY, Wang P. SP1-mediated upregulation of lncRNA SNHG4 functions as a ceRNA for miR-377 to facilitate prostate cancer progression through regulation of ZIC5. J Cell Physiol. 2020;235 (4):3916-3927. doi:10.1002/jcp.29285

33. Ponomarev ED, Veremeyko T, Weiner HL. MicroRNAs are universal regulators of differentiation, activation, and polarization of microglia and macrophages in normal and diseased CNS. Glia. 2013;61 (1):91-103. doi:10.1002/glia.22363

34. Ponomarev ED, Veremeyko T, Barteneva N, Krichevsky AM, Weiner HL. MicroRNA-124 promotes microglia quiescence and suppresses EAE by deactivating macrophages via the C/EBP-alpha-PU.1 pathway. Nat Med. 2011;17(1):64-70. doi:10.1038/nm.2266

35. Xu R, Zhao M, Yang Y, et al. MicroRNA-449c-5p inhibits osteogenic differentiation of human VICs through Smad4-mediated pathway. Sci Rep. 2017;7(1):8740. doi:10.1038/s41598-017-09390-z

36. Jiang L, Wu C, Wu J, Yang X, Wang X,H, Li G. Microarray-based measurement of microRNA-449c-5p levels in hepatocellular carcinoma and bioinformatic analysis of potential signaling pathways. Pathol Res Pract. 2019;215(1):68-81. doi:10.1016/j.prp.2018.10.007

37. Lawrence T, Natoli G. Transcriptional regulation of macrophage polarization: enabling diversity with identity. Nat Rev Immunol. 2011;11(11):750-761. doi:10.1038/nri3088

38. Hou J, Schindler U, Henzel WJ, Ho TC, Brasseur M, McKnight SL. An interleukin-4-induced transcription factor: IL-4 Stat. Science (New York, N Y). 1994;265(5179):1701-1706. doi:10.1126/science.8085155

39. Czimmerer Z, Daniel B, Horvath A, et al. The transcription factor STAT6 mediates direct repression of inflammatory enhancers and limits activation of alternatively polarized macrophages. Immunity. 2018;48(1):75-90.e6. doi:10.1016/j.immuni.2017.12.010

40. Wang Q, Wei S, Zhou $\mathrm{H}$, et al. Hyperglycemia exacerbates acetaminophen-induced acute liver injury by promoting liver-resident macrophage proinflammatory response via AMPK/ PI3K/AKT-mediated oxidative stress. Cell Death Discovery. 2019;5:119. doi:10.1038/s41420-019-0198-y

\section{Publish your work in this journal}

Drug Design, Development and Therapy is an international, peerreviewed open-access journal that spans the spectrum of drug design and development through to clinical applications. Clinical outcomes, patient safety, and programs for the development and effective, safe, and sustained use of medicines are a feature of the journal, which has also been accepted for indexing on PubMed Central. The manuscript management system is completely online and includes a very quick and fair peer-review system, which is all easy to use. Visit http://www. dovepress.com/testimonials.php to read real quotes from published authors. 